\title{
Hermann-Altrock-Stipendium des Deutschen Sportbundes
}

\section{Ausführungsbestimmuugen}

1. Das Hermann-Altrock-Stipendium wird alljährlich in Höhe von DM 5000,- an einen Sportstudierenden ader einen Doktoranden aus der Bundesrepublik Deutschland verliehen.

2. Das Stipendium soll es dem Empfänger ermöglichen, nach bestandener sportpraktischer Prüfung sein Studium für zwei Semester im Ausland fortzusetzen bzw. eine thematisch wichtige sportwissenschaftliche Dissertation anzufertigen.

3. Vorschläge für die Verleihung mit Refenenzen der Hochschullehrer und einem handschriftlichen Lebenslauf des Bewerbers (mit Lichtbild), in dem dieser u. a. die beabsichtigte Verwendung des Stipendiums darlegt, sind durch die Institute für Leibesübungen und die Pädagogischen Hochschulen jeweils zum 1. Oktober an den

$$
\begin{aligned}
& \text { Deutschen Sportbund, } \\
& \text { Bundesausschuß für Wissenschaft und Bildung, } \\
& \text { Otto-Fledk-Schneise 12, } \\
& 6000 \text { Frankfurt am Main 71, }
\end{aligned}
$$

zu richten.

4. Beurteilungskriterien für die Vergabe des Stipendiums sind

- das Abiturzeugnis

- die Ergebnisse der Ersten Staats-(Lehrer-)Prüfung bzw. ihres praktischen Teils,

- Zeugnisse aus anderen Studienfächern,

- Thema und Aufbau der geplanten Dissertation,

- die Mitarbeit im Studium, insbesondere in Seminaren und an Forschungsvorhaben,

- die Bewährung bei der Leitung von Jugendgruppen und/oder die Mitwirkung in Turn- und Sportvereinen.

5. Die Entscheidung über die eingereichten Vorschläge trifft der Bundesausschuß für Wissenschaft und Bildung im Benehmen mit dem Wissenschaftlichen Beirat des DSB. 